\title{
Subra Philippe, Zones à Défendre, de Sivens à Notre- Dame-des-LandesL'Aube, 2016, 120 p.
}

\section{Philippe Boulanger}

\section{OpenEdition}

Journals

Édition électronique

URL : https://journals.openedition.org/geohist/1420

DOI : $10.4000 /$ geohist. 1420

ISSN : 2264-2617

\section{Éditeur}

Association française de la Revue de géographie historique

\section{Référence électronique}

Philippe Boulanger, «Subra Philippe, Zones à Défendre, de Sivens à Notre-Dame-des-

LandesL'Aube, 2016, 120 p. », Revue de géographie historique [En ligne], Comptes-rendus, mis en ligne le 20 novembre 2016, consulté le 12 juin 2021. URL : http://journals.openedition.org/geohist/1420 ;

DOI : https://doi.org/10.4000/geohist.1420

Ce document a été généré automatiquement le 12 juin 2021.

\section{c) (i) $\Theta$}

Ce(tte) œuvre est mise à disposition selon les termes de la Licence Creative Commons Attribution Pas d'Utilisation Commerciale - Pas de Modification 4.0 International. 


\title{
Subra Philippe, Zones à Défendre, de Sivens à Notre-Dame-des- LandesL'Aube, 2016, 120 p.
}

\author{
Philippe Boulanger
}

\section{RÉFÉRENCE}

Subra Philippe, Zones à Défendre, de Sivens à Notre-Dame-des-Landes,

L'Aube, 2016, 120 p.

1 Le référendum du 26 juin 2016 pour le Grand Aéroport de Notre-Dame-des-Landes, en Loire-Atlantique, où le oui l'emporte à $55,17 \%$ des voix, donne à comprendre une situation complexe. Qu'est-ce qu'une ZAD? Il est devenu difficile de définir ce phénomène présenté dans les médias sous le nom de ZAD (Zone A Défendre), mais aussi de zone grise ou de zone de non droit, expressions réservées en géopolitique pour désigner des territoires devenus incontrôlables, voire de guerre civile comme en Somalie dans les années 2000 ou en Afghanistan avant 2001. Philippe Subra, spécialiste de la géopolitique locale et de l'aménagement du territoire à l'Institut français de géopolitique (Université Paris VIII), auteur de plusieurs ouvrages de référence en la matière, nous livre un essai de réflexion très éclairant sur ce phénomène de société protéiforme à partir des principaux projets actuels (comme Sivens, Bure, NotreDame-des-Landes, Roybon, les ZAD 2.0) en France, tout en le mettant en perspective avec des mouvements de contestations passés (comme le projet d'extension du camp militaire du Larzac dans les années 1970). Si l'ouvrage est éclairant, c'est que l'auteur offre des comparaisons avec les nombreux mouvements d'opposition contre des projets d'aménagement locaux en France depuis les années 1960 à travers le prisme du géographe, c'est-à-dire de l'analyse réfléchie et concrète des rivalités de pouvoirs entre des acteurs différents sur un territoire donné. Il montre de manière démonstrative la spécificité du zadisme qui s'est développé par la «convergence entre un altermondialisme 
qui se territorialise et une concertation environnementale qui se radicalise " remettant en cause le processus de concertation établi entre les acteurs depuis plusieurs décennies.

Le plan de l'ouvrage pose d'ailleurs des questions essentielles auxquelles Philippe Subra répond de manière précise et synthétique : 1) Qu'est-ce qu'une ZAD, 2) d'où viennent les ZAD ?, 3) Demain, les ZAD partout? 4) Pour les aménageurs et pour l'Etat, le referendum est-il le moyen de sortir du piège ? L'ouvrage est très instructif pour $\mathrm{y}$ trouver des repères, comprendre les évolutions du mouvement zadiste, évaluer ses enjeux et ses évolutions sociétales, mesurer la place du territoire dans ses affrontements devenus plus violents. Il montre, en particulier, que le développement et la pérennité d'une ZAD reposent sur trois conditions majeures : un projet contre lequel la population locale est unie dans l'opposition mais dont les formes classiques de lutte ont été vaines, un terrain favorable par sa topographie et son milieu naturel (le bocage par exemple) favorisant sa défense, la proximité d'une grande ville, généralement estudiantine, qui apporte son soutien au mouvement de contestation, la capacité d'alliance entre les zadistes et les opposants locaux. La ZAD de Notre-Dame-des-Landes, selon Philippe Subra, est exemplaire: "Les objectifs stratégiques des zadistes et des opposants classiques (...) ne coïncident que partiellement. Les modes d'action des opposants classiques et zadistes different également, mais cette diversité est perçue comme une complémentarité (...). Les intérêts qu'ont en commun les opposants à la création de l'aéroport, zadistes et opposants classiques, ont permis jusqu'ici de dépasser ces différences (...)».

3 L'ouvrage nous apprend beaucoup sur l'évolution du mouvement altermondialiste qui connaît une mutation majeure en donnant lieu au Zadisme. Il montre, d'abord, que celui-ci devient "une mutation stratégique d'un mouvement à la recherche de combats concrets» dont la territorialisation prend un sens nouveau. Le territoire n'est plus urbain mais rural, plus structuré en réseau et enraciné qu'éphémère à l'occasion d'un grand sommet du G7. Il apparaît aussi réticulaire à différentes échelles géographiques : au niveau local (la ZAD), au niveau régional (l'espace rural hors ZAD, la relation avec les villes voisines), au niveau national voire européen (avec l'ensemble des ZAD). Au-delà des territoires, le mouvement évolue pour durer, définir des objectifs stratégiques en lien avec les opposants anciens, conduire des actions diluées mais aussi plus violentes par rapport aux mouvements antérieurs (Larzac par exemple).

Philippe Subra analyse également par quels moyens le zadisme devient un mouvement de luttes environnementales aux formes radicalisées. La seule issue du mouvement est la victoire contre des projets perçus comme néfastes pour la démocratie et la Planète dans un contexte de constat d'impuissance du parti écologiste. "La violence est redevenue une option, alors qu'elle était absente des mouvements de contestation des projets d'aménagement depuis le début des années 1980 ». Enfin, les modes de concertation entre les différents acteurs sont bousculés par la nouvelle culture d'opposition du zadisme et par la radicalisation de ses modes d'actions. Tout en rappelant le succès de plus de 80 débats publics en France entre 1997 et 2016, les progrès de l'information auprès des populations dans le cadre des projets d'aménagements, permettant de réduire les conflits locaux, l'auteur souligne la difficulté de mener des opérations de dialogue et de négociation avec un mouvement qui se radicalise. Ce dernier point demeure un enjeu fondamental dans les prochains mois et les prochaines années pour la société et l'Etat.

5 Aux termes de la lecture de cet essai, force est de constater la qualité d'analyse et de démonstration. Il s'agit d'un livre sans nul doute à découvrir et à relire pour mesurer la dimension nouvelle et protéiforme d'un mouvement inscrit dans la durée. 